\title{
Influence of bacteria, diffusion and shear on micro-scale nutrient patches, and implications for bacterial chemotaxis
}

\author{
Nicholas Blackburn*, Tom Fenchel \\ Marine Biological Laboratory, University of Copenhagen, Strandpromenaden 5, 3000 Helsingør, Denmark
}

\begin{abstract}
Micro-scale nutrient patches were observed in mixtures of isolates consisting of a protozoan, its prey, and chemotactic bacteria. The patches were shown by swarms of bacteria and the events leading to patches were associated with cell lysis and predation events. In such a form, nutrients are experienced by bacteria as pulses. Simulations showed that patches can be consumed by the bacterial community before being dispersed. As a result, even non-motile bacteria may only ever encounter diffuse patches within a sphere of $2 \mathrm{~mm}$ radius. If patches are generated randomly in time and space, it can be advantageous for a bacterium to swim, if only to break this $2 \mathrm{~mm}$ barrier. Simulations suggested that chemotaxis can increase a bacterium's exposure to nutrients within a patch, but that the enhancement depends on the size of the patch, due to limitations in sensitivity. The patch size limit for achieving enhancement is $\sim 0.1$ pmol. Swimming speed is the most important motility parameter influencing efficiency of chemotactic foraging on diffuse patches, with an optimum speed as a function of patch size. Simulations illustrated that shear stretches patches, but that moderate shear does not significantly alter patch volumes within time scales of several minutes. A large proportion of the nutrients within a patch can be encountered by bacteria within that time.
\end{abstract}

KEY WORDS: Bacteria $\cdot$ Micro-scale patchiness $\cdot$ Chemotaxis $\cdot$ Shear

\section{INTRODUCTION}

There are technical difficulties in measuring microscale distributions of dissolved organic and inorganic nutrients. Proof of their existence lies in observations of the behavior of bacterial swarms (Blackburn et al. 1998). Recorded patchiness in microbial communities is also a strong indication (Duarte \& Vaqué 1992, Krembs et al. 1998), but does not offer direct proof, because it can be the result of e.g. colonization of transparent exopolymers. Definite sources attracting bacterial swarms have been identified as cell lysis and the emptying of food vacuoles by protists (Blackburn et al. 1998). These processes are important pathways in the recycling of nutrients for growth in the microbial food web. It makes sense that bacteria, which are adapted to make use of dissolved nutrients, should also

•E-mail: mblnb@mail.centrum.dk be adapted to optimizing their exposure to them. The patches mentioned were shown to correspond to near point source releases of nutrients in quantities of the order of 1 pmol, which subsequently spread by molecular diffusion. Here we present a method for observing similar phenomena under more controlled conditions by mixing isolates of microbes in small observation chambers.

A number of interesting questions arise from a theoretical point of view, given that important nutrients are regenerated through point source events: What would the growth pattern of bacteria look like? To what degree does water movement influence patches? What gain in growth could be expected with chemotaxis? How is bacterial swimming behavior adapted for foraging on patches? We have used simulations to answer important aspects of these questions, based on a simple model of chemotaxis and basic models of the physical processes involved in patch dispersal and consumption. 


\section{METHODS}

Mixtures of organisms. Seawater was enriched with tryptic soy broth (ca $0.1 \%$ ) and left for $24 \mathrm{~h}$. High concentrations of motile bacteria grew in the culture. Residual growth medium was diluted by twice centrifuging $(5000 \times g)$ and re-suspending the pellet (Adler 1973). Final concentrations of bacteria were ca $10^{7} \mathrm{ml}^{-1}$. A pure culture of Rhodomonas salina was treated in the same way at $500 \times g$ and added to the bacterial culture, so that the concentration of $R$. salina was ca $10^{5} \mathrm{ml}^{-1}$. The centrifuging had no apparent effect on swimming behavior. A pure culture of the dinoflagellate Oxyrrhis marina, grown on $R$. salina, was added to a portion of the mixture, so that $O$. marina was present at a concentration similar to $R$. salina. O. marina began to capture and ingest $R$. salina immediately after being introduced. The final mixture was left overnight.

Flat glass capillaries $(4 \times 0.4 \mathrm{~mm}$ cross section, CamLab, United Kingdom ] were used as observation chambers. A $20 \times$ objective was used on a standard microscope fitted with a dark-field condenser. The depth of field was ca $20 \mu \mathrm{m}$ for bacteria, but deeper for the larger cells. The light bulb of the microscope was replaced with the tip of a fiber optic lamp to reduce heat transfer to the chambers. A standard video camera and VCR were used for recordings, and a black and white frame grabber card (IMAQ $1408^{\text {TM }}$, National Instruments) was used to digitize sequences in real time (PAL format, 25 frames $\mathrm{s}^{-1}$, half resolution) to computer memory. The sequence was then analyzed frame by frame for trajectories of movement by LabTrack $^{\text {rM }}$ (DiMedia, Denmark).

Poisson patches. The effect of patches occurring randomly in time and space was analyzed by simulation. Bacteria and algae were evenly distributed in 3dimensional space at given densities. Algal cells were represented explicitly at fixed locations in space. Algae were the sources of patches (in real life as a result of predation and autolysis) occurning randomly in time. This was simulated using a random generator to determine whether or not a given cell should result in a patch within the simulated time step dt, resulting in a Poisson process. The probability $P$ of such an event occurring was calculated as

$$
P=\frac{G}{\mathrm{~d} t}
$$

where $\mathrm{d} t$ is the time step, and $G$ is average life span corresponding to the turnover time of the algal population (as would be the case in steady state). This calculation was performed for each algal cell. Each time a patch was created, the starting time for the event was recorded, and its contribution to the total concen- tration at the origin could be calculated as a function of time after the start of the event and its fixed distance from the origin. In order to do this, the concentration of matter in a patch was pre-calculated as a function of time and distance from its source, under the influence of diffusion limited uptake by the bacterial population at its given density. The function was stored in a look-up table. The table was constructed by running a simulation similar to that described in Blackburn et al. (1997) using LabMatic ${ }^{\text {TM }}$ (DiMedia, Denmark), where matter was allowed to diffuse from a point source across concentric spherical shells. The gross uni-directional mass flow $F$ across the interface between 2 shells was approximated from Fick's first law of diffusion as

$$
F=D \frac{r}{\mathrm{~d} r^{2}}
$$

where $D$ is the diffusion coefficient $\left(10^{-5} \mathrm{~cm}^{2} \mathrm{~s}^{-1}\right), r$ is the radial distance of the shell from the source, and $\mathrm{d} r$ is the thickness of the shell. The table extended from 0 to $5000 \mu \mathrm{m}$ in steps of $50 \mu \mathrm{m}$ and 0 to $4000 \mathrm{~s}$ in steps of $50 \mathrm{~s}$. The consumption $U$ of matter within a shell was assumed to be diffusion limited and was calculated as

$$
U=B V 4 \pi a D C
$$

where $a$ is the radius of a bacterium $(0.25 \mu \mathrm{m}), B$ is the density of bacteria $\left(3 \times 10^{5} \mathrm{ml}^{-1}\right), V$ is the volume of the shell, and $C$ is the concentration of matter in the shell. The resulting sum of contributions from all patches at the origin represented a typical timedependent concentration field at any point in space, resulting from randomly occurring patches from evenly distributed sources, under the influence of consumption by evenly distributed consumers. The assumption of diffusion limitation is realistic (Koch \& Wang 1982) and convenient, because it allows growth to be defined in terms of a simple physical constraint (Jumars et al. 1993).

The background concentration $C$ of nutrients assuming steady state is given by

$$
C=\frac{S}{B 4 \pi a D G}
$$

where $S$ is the density of sources $\left(1000 \mathrm{ml}^{-1}\right)$. The concentration $C$ is in units of source mass $\mathrm{ml}^{-1}$.

Simulation of chemotaxis. Bacteria move in a biased random walk comprised of runs interspersed with tumbles or reversals. The model of chemotaxis developed for the enteric bacterium Escherichia coli (Brown \& Berg 1974) was shown to apply to some faster-swimming marine bacteria (Blackburn et al. 1998). The probability $P$ of an individual tumbling within a small simulated time step $\mathrm{d} t$ is 


$$
P=\frac{\mathrm{d} t}{\tau}
$$

where $\tau$ is the mean run duration. A random generator was used to decide the outcome (tumble or no tumble) for each time step; in effect, simulating the desired Poisson process (Berg \& Brown 1972). The probability of tumbling was decreased, when running up a gradient, by increasing the mean run duration with the term $\Delta \tau$

$$
\Delta \tau=\frac{\alpha}{k_{\mathrm{D}}} \frac{\partial C}{\partial t}
$$

where $\alpha$ is a sensitivity constant (1000 s), $k_{\mathrm{D}}$ is a dissociation constant $(100 \mu \mathrm{M})$, and $C$ is the concentration of attractant. The rate of change of attractant concentration was calculated as the difference between concentration at the start and end of a run over a period of $\mathrm{d} t$, at a given velocity and heading direction (Bowen et al. 1993, Jackson 1987, Blackburn et al. 1998). A random rotation of $1 \mathrm{rad} \mathrm{s}^{-1}$ was also introduced to simulate Brownian rotation. Simulations were performed in 2 dimensions relative to the 3 -dimensional concentration field for a patch spreading from a point source given by

$$
C=(4 \pi D t)^{-1.5} \exp \left(r^{2} / 4 D t\right)
$$

where $t$ is time and $r$ is the radial distance from the source. Individuals were initially positioned at random within a radius of $1 \mathrm{~mm}$ from the source.

Simulation of diffusion, shear, and consumption. The method used is described in Blackburn et al. (1987) using a 2-dimensional grid. Diffusion is the transfer of mass between neighboring grid cells and consumption is the removal of mass from each grid cell. Consumption was calculated using Eq. (3), but with the volume of a grid cell rather than a shell. Bacterial density was set to $10^{6} \mathrm{ml}^{-1}$. In addition, shear was added as a uni-directional linear velocity gradient (aligned with the $y$-axis of the grid) which is a good approximation to reality at these small spatial scales (<3 mm) (Lazier \& Mann 1989, Bowen \& Stolzenback 1992, Bowen et al. 1993). The rate of dispersion $A$ of mass in a given grid cell is

$$
A=m \mathrm{~d} x \Omega y
$$

where the shear rate is given by $\Omega, m$ is the mass in the cell, $y$ is distance from the point of reference where the relative velocity is 0 (in this case the center of the grid), and $\mathrm{d} x$ is the length of a grid cell in the direction of the $x$-axis. This method of simulation introduces a secondary, artificial dispersion along the $x$-axis, but it is of negligible importance in this context. It is common to choose a value for the shear rate $\Omega$ (here $0.05 \mathrm{~s}^{-1}$ ) which is a maximum for a given energy dissipation rate $\varepsilon(\Omega=$ $0.05 \mathrm{~s}^{-1}$ corresponds to $\varepsilon \approx 10^{-8} \mathrm{~W} \mathrm{~kg}^{-1}$ ), which characterizes the system (Lazier \& Mann 1989). These values represent moderate shear and turbulence conditions.
The 2-dimensional case was chosen for computational simplicity. It suffices for the purpose of calculating patch life and dispersion. This is because dispersion occurs at the same rate as in 3 dimensions, whereas it is the concentration that differs between the cases. The effect on uptake of higher concentrations (higher uptake rates) in the 2-dimensional case, however, exactly cancels out against the correspondingly lower bacterial numbers in the 3-dimensional case, because uptake is proportional to bacterial density and nutrient concentration.

\section{RESULTS}

\section{Mixtures of organisms}

Swarms of bacteria around dead cells of both Rhodomonas salina and Oxyrrhis marina resembling the example given by Blackburn et al. (1998) (their Fig. 1A) were observed under all conditions. No obvious source of attractant was evident for some of the swarms (Fig. 1A). These were only observed in mixtures including the predator (O. marina), and we thus assume that the sources of these patches were associated with incomplete assimilation on the part of the predators. O. marina also displayed chemotactic behavior (Fig. 1B). We believe that they were responding to the same chemical gradient as the bacteria, because they homed in on the exact center of patches before quickly leaving them again.

\section{Diffusion, shear, and consumption}

Uni-directional shear stretches patches into ellipsoids. At moderate shear rates and with the given parameters, the ellipsoid has a length of ca $26 \mathrm{~mm}$ and is ca $2 \mathrm{~mm}$ in diameter after $300 \mathrm{~s}$, corresponding to a volume of $54 \mathrm{~mm}^{3}$. The equivalent result in the absence of shear is a patch of ca $4 \mathrm{~mm}$ in diameter, corresponding to a volume of $35 \mathrm{~mm}^{3}$ (Fig. 2). The patches in both cases were reduced to $40 \%$ in mass by diffusion limited uptake within $300 \mathrm{~s}$ at a bacterial density of $10^{6} \mathrm{ml}^{-1}$

\section{Poisson patches}

The simulation showed that a bacterium experiences patches as pulses (Fig. 3). The time averaged concentration over several simulations (representing several days) was approximately normally distributed with a mean of 6 units of source mass $\mathrm{ml}^{-1}$ and an equivalent standard deviation. Background levels were 1.5 units 


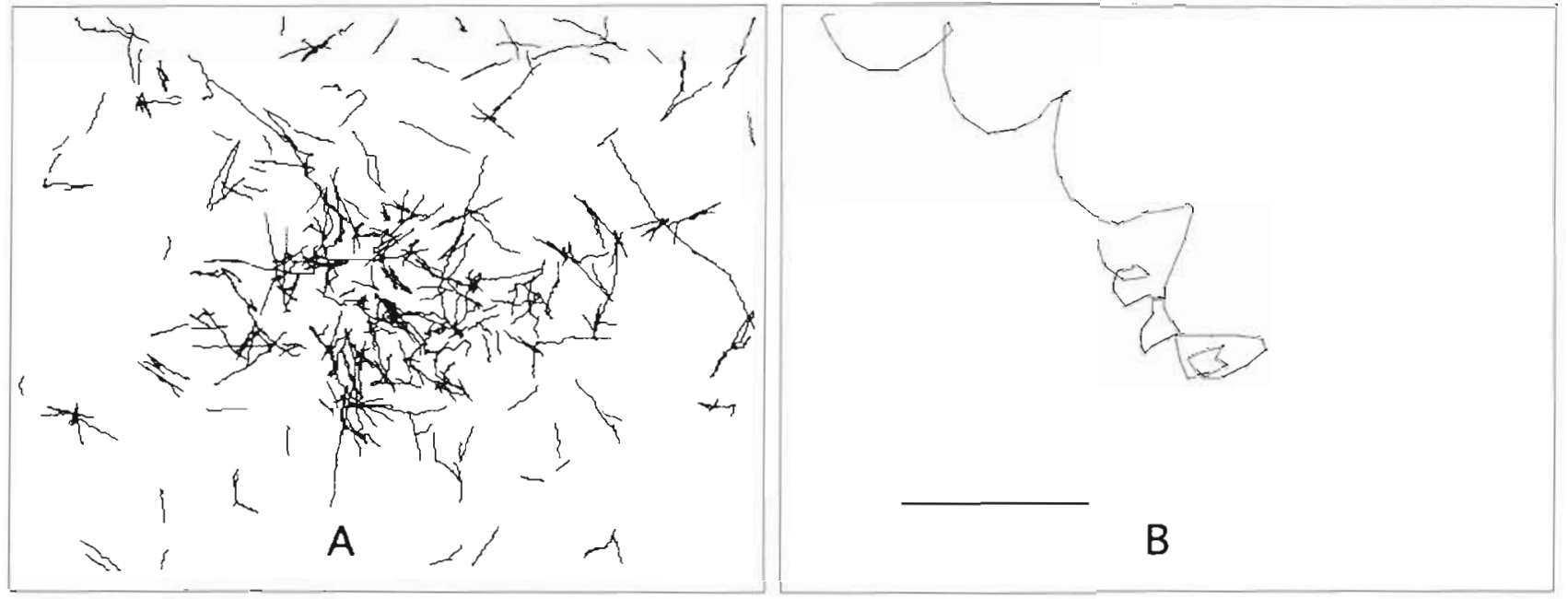

Fig. 1. Tracks of motile micro-organisms responding to a diffuse patch. (A) Bacteria swimming at $50 \mu \mathrm{m} \mathrm{s}^{-1}$ on average and with run durations of $0.45 \mathrm{~s}$. The concentration in the patch is ca $1.5 \times 10^{7}$ (B) The track of a single Oxyrrhis marina responding to the same patch. Time between points on tracks is $0.04 \mathrm{~s}$. Bar $100 \mu \mathrm{m}$

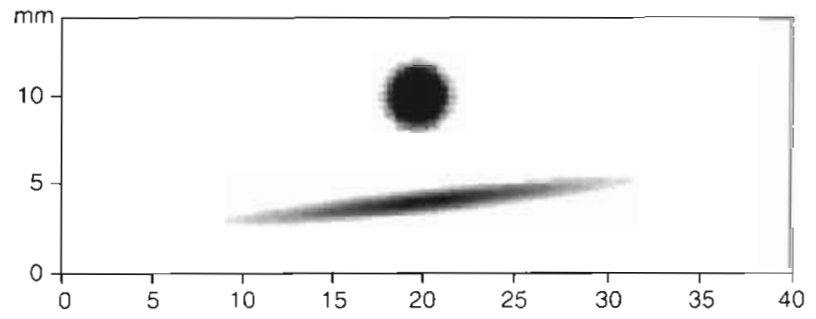

Fig. 2. Simulations of patches originating from point sources in the presence of uni-directional, steady shear at $0.05 \mathrm{~s}^{-1}$ and in the absence of shear, over a time period of $300 \mathrm{~s}$ and influenced by diffusion limited consumption by bacteria at a density of $10^{6} \mathrm{ml}^{-1}$. Resolution was $0.5 \mathrm{~mm}$. Spatial scale in $\mathrm{mm}$

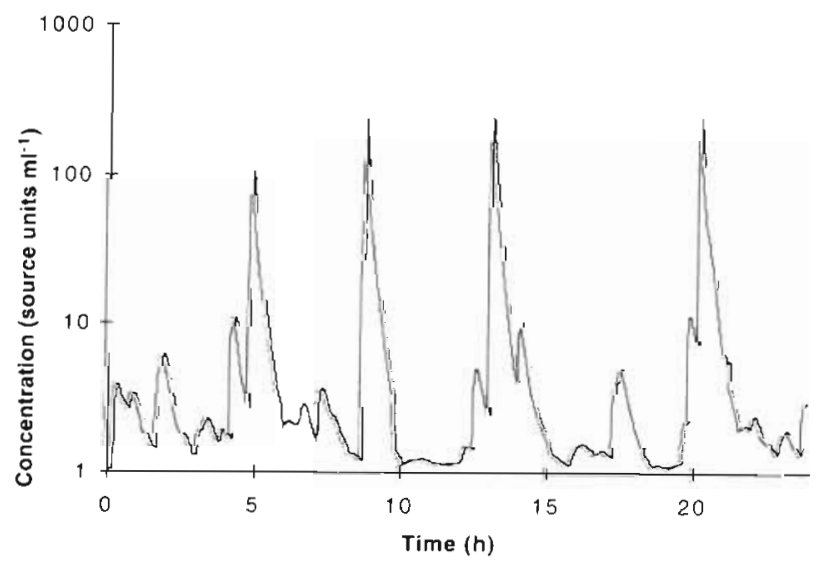

Fig. 3. Simulations of nutrient concentration with time at an arbitrary point in space, originating from evenly distributed sources at a density of $10^{3} \mathrm{ml}^{1}$, with turnover times of $24 \mathrm{~h}$. Concentration is given in units of source mass $\mathrm{ml}^{-1}$ of source mass $\mathrm{ml}^{-1}$ (a quarter of the mean). The case where nutrients are immediately and homogeneously mixed gives a background concentration in steady state of 10.6 units of source mass $\mathrm{ml}^{-1}$ with the given parameters (Eq. 4). The mean was lower in the simulations, due to approximations made as a result of the finite resolution of the look-up table.

\section{Bacterial chemotaxis}

Simulations illustrate different swarm behavior as a function of patch size (i.e. the amount of matter in the patch). The smaller the patch, the smaller the sphere within which bacteria will be able to respond, and the quicker the patch will disperse below sensitivity levels (Fig. 4). The expected gain in nutrient encounter achieved by chemotaxis for a bacterium placed within a radius of $1 \mathrm{~mm}$ from the source in the 3 situations shown in Fig. $4 \mathrm{~A}$ to $\mathrm{C}$ was 15, 50, and $75 \%$, respectively.

The analysis of the importance of different motility parameters over a period of $10 \mathrm{~min}$ revealed that velocity was the most influential parameter contributing to increasing nutrient exposure by chemotaxis (Fig. 5). In this particular example of a spreading patch of mass 1 pmol, the possible average gain was 2.5. A decreasing ability to maneuver close to the target, with velocities exceeding $300 \mu \mathrm{m} \mathrm{s}^{-1}$, resulted in decreasing gain at higher velocities. There was a negative gain at small turn angles because of an effective dispersion away from the source, but turn angles exceeding $90^{\circ}$ did not result in significant gain (a turn angle of $90^{\circ}$ corresponds on average to random orientation after tum- 
A

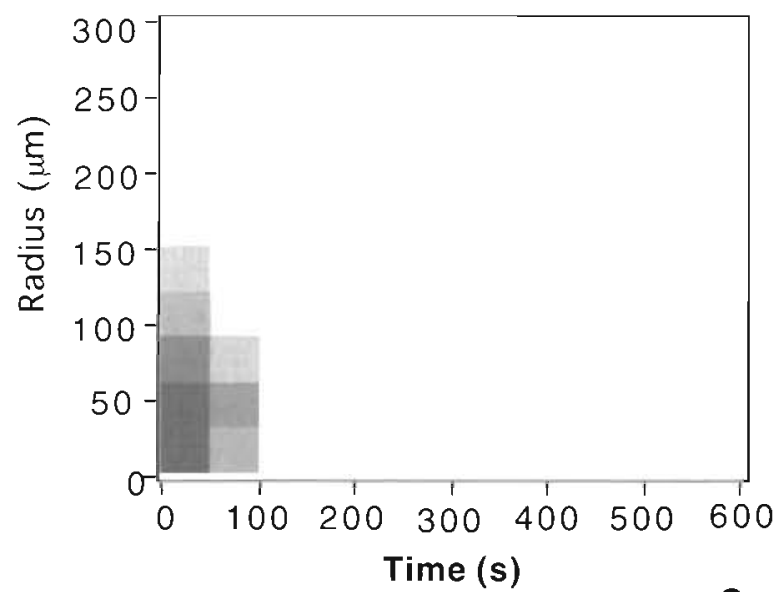

C

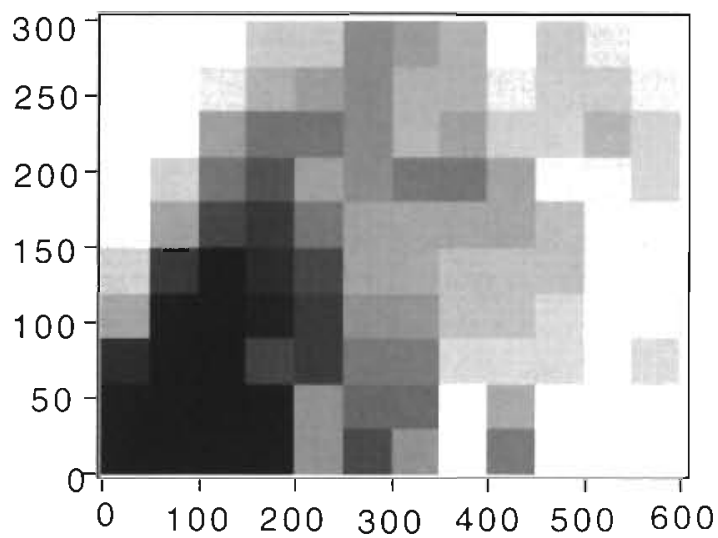

B

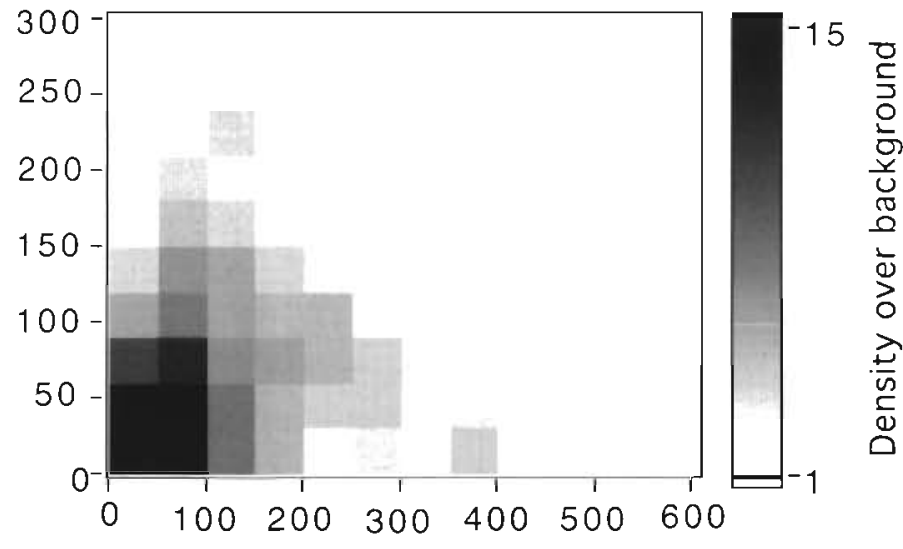

D

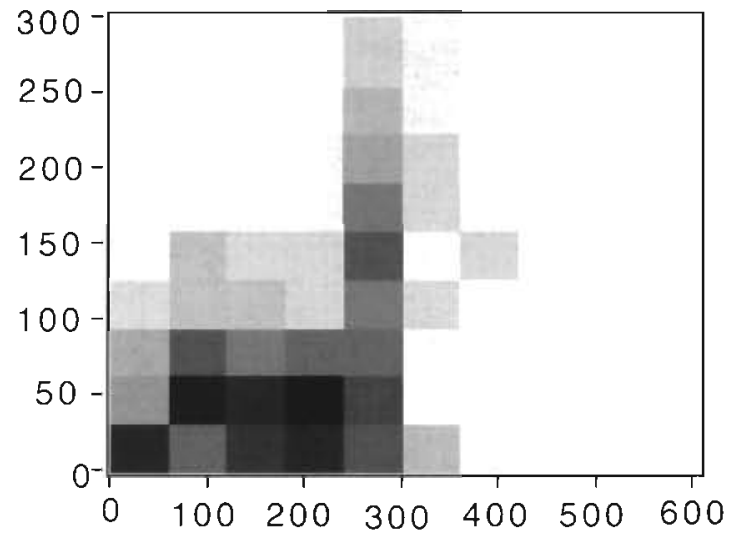

Fig. 4. Simulations of chemotactic bacterial swarm density as a function of radius and time responding to a point source spreading by molecular diffusion. The density is normalized to the background. Swimming velocity $50 \mu \mathrm{m} \mathrm{s}^{-1}$, run duration $0.4 \mathrm{~s}$. (A) Point source release of 0.05 pmol. (B) 0.2 pmol. (C) 1 pmol. (D) The equivalent analysis of the swarm which formed around a lysed ciliate shown in Blackburn et al. (1998), Fig. 1A

bling). It appeared to be marginally advantageous to tumble often i.e. to swim with short mean run durations, but run durations exceeding $0.4 \mathrm{~s} \mathrm{had} \mathrm{only} \mathrm{a} \mathrm{very}$ slight negative effect on the gain. Brownian rotation generally had a negative effect on gain, but bacteria rotating as quickly as $10 \mathrm{rad} \mathrm{s}^{-1}$ were still able to achieve some advantage.

\section{DISCUSSION}

The present observations of mixed communities of microbes under relatively controlled conditions showed similar phenomena to those observed in in situ samples from a biofilm (Blackburn et al. 1998), namely, that swarms of bacteria appear around lysed cells and clouds with no visible sources. The present experiments support the assumption that it is incomplete digestion on the part of predators that acts as sources of such patches. The behavior of bacteria observed here resembled that displayed by 2 morphologically distinct species of bacteria from a biofilm (Blackburn et al. 1998), which suggests a degree of predictability in the response of bacterial swarms to diffuse patches. The model of chemotaxis fits the observed responses of bacterial swarms, which in turn fits the assumption that the attractant is present in a patch spreading by molecular diffusion (Blackburn et al. 1998) (Fig. 4D). The examples of patch-generating events mentioned here represent important pathways for the regeneration of nutrients in the microbial food web (Azam \& Ammerman 1984, Blackburn et al. 1998, Krembs et al. 1998), but there are probably a number of other mechanisms that generate patches. Some might occur on longer time scales, and would resemble exudation processes. 


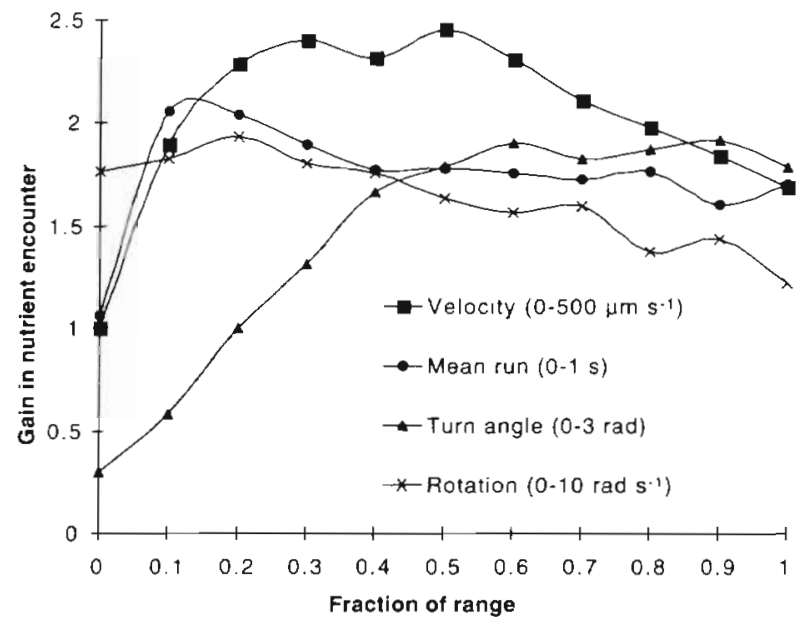

Fig. 5. Analysis by simulation of the importance of different swimming parameters, velocity, run duration, turn angle, and Brownian rotation, on the mean nutrient exposure gain achieved by swimming in a diffuse patch. Each point is the result of a simulation of 1000 bacteria over $500 \mathrm{~s}$, where each parameter was changed, in turn, over a defined range. Bacteria were initially randomly distributed within a sphere of 1 $\mathrm{mm}$ radius from the source. Swimming speed was $50 \mu \mathrm{m} \mathrm{s}^{-1}$ and run duration was $0.4 \mathrm{~s}$ in the basic model. The point source of attractant was 1 pmol

An important question is: What proportion of limiting nutrients are released as near point sources under specific conditions? This is not easy to answer given current technology. A large proportion could potentially be involved, because most relevant events involve individual cells, which represent point sources. Ideally, in order to verify this, fluorescent probes of some type might be developed for visualizing dissolved pools. It is possible to look for clusters of bacteria which indirectly indicate nutrient patchiness (Krembs et al. 1998), but the absence of such clusters does not rule out the presence of nutrients in patches. For example, a nutrient patch has to be of a certain size for an associated bacterial swarm to become clearly visible (Fig. 4), and swarming cannot be expected under all conditions, especially when organism sizes and patch sources are small.

The importance of micro-scale patches depends somewhat on perspective. However, even from a global point of view, they can have significant implications for the flow of energy and nutrients in the oceans. On time scales of hours, the transfer of dissolved compounds into the food chain can be greatly accelerated (Fig. 3). Even though the gross flow might not differ from the completely mixed case, the transfer rates differ greatly, and this has unpredictable effects on the food chain as a whole. In the simulated example presented here, patchiness also resulted in background concentrations much lower than those in the mixed case. From the point of view of an individual bacterium, patches represent resources that are present within limited time and space, and it becomes a challenge to optimize foraging under such conditions; swimming is a means of achieving this.

Observations of low swimming speeds in pelagic environments are consistent with the expectation of small patch sizes, because high swimming speeds are not advantageous under such conditions (Fig. 5). In contrast, enriched samples, sediments, and biofilms typically contain some strains of much faster bacteria (Barbara \& Mitchell 1996, Mitchell et al. 1996, Fenchel \& Glud 1998). We suggest that this behavior is adapted for encountering larger patches occurring less frequently in time and space.

Assuming that the fate of primary producers in a microbial food web results in point source release of nutrients, the patches are experienced by bacteria as pulses (Fig. 3). The result is influenced by the bacterial density and their uptake potential. The pulses directly reflect the growth rate. Stochasticity in patch occurrence also introduces day to day variability, e.g. there was a $15 \%$ chance of encountering twice (or half) the daily average on any particular day. The simulation showed that although 1000 patches were generated per day within a volume of $1 \mathrm{ml}$, only approximately 20 $\left(\sim 1 \mathrm{~h}^{-1}\right)$ were ever experienced by any particular bacterium (the number occurring within a radius of $2 \mathrm{~mm}$ ). This points to the possible importance of swimming for encountering patches, in addition to foraging on them upon encounter. A significant gain in patch encounter would be achieved by swimming fast enough to break this $2 \mathrm{~mm}$ barrier (i.e. $2 \mathrm{~mm}$ in less than $-1 \mathrm{~h}$ ). A speed of just $10 \mu \mathrm{m} \mathrm{s}^{-1}$ is enough to propel a bacterium $2 \mathrm{~mm}$ in -20 min if it is moving in a random walk. If this hypothesis is accurate, it can offer an explanation for very slow swimming velocities typical for bacteria in pelagic environments.

Under normal circumstances dispersion by turbulence does not occur at spatial scales below $1 \mathrm{~mm}$ (Lazier \& Mann 1989). There is, however, a shear which results in stretching, as shown in Fig. 2. Often the stretching occurs uni-directionally, but it can also occur in 2 dimensions, resulting in disc-like structures (Bowen \& Stolzenback 1992, Bowen et al. 1993). Turbulence results in increasingly diverging directions of water movement with distance, so the patch shown in Fig. 2 would become distorted, especially at its extremities. The simulation illustrated that the shear reduces dispersion by diffusion. This can be seen in Fig. 2, where the dispersion of matter along the velocity gradient (vertical direction) in the patch is much lower in shear flow. The volume of the patch in this example of moderate, steady shearing was only twice that of a patch spreading in the absence of shear. The result 
depends on the density of bacteria and their uptake potential, as it did in the case of randomly generated patches in space and time, and the given example should be considered a representative visualization of the effect of shear. A study in Monterey Canyon found that the water mass contained shear layers several meters in thickness (Itsweire \& Osborn 1988). The average shear rate within one of the turbulent layers was estimated to be $0.02 \mathrm{~s}^{-1}$. The maximum shear exceeded the average only periodically, and the time scale for fluctuations was minutes. There appeared to be extended areas in space and/or periods in time of much lower shearing, even within the layers. These observations, together with the simulation, indicate the potential for stability of micro-scale diffuse patches under a wide range of conditions in aquatic systems.

We have hereby outlined a number of challenges for future research in microbial ecology. It is relatively easy to observe and record motility patterns of individual bacteria, but there is scope for improving visualization techniques. There is very much more to be discovered about bacterial foraging strategies in nature and the constraints which are set by the structure of the physical micro-environment.

Acknowledgements. We thank the Swedish Foundation for International Cooperation in Research and Higher Education (STINT), and the Danish National Research Council (SNF) for support of this study.

\section{LITERATURE CITED}

Adler J (1973) A method for measuring chemotaxis and use of the method to determine optimum conditions for chemotaxis by Escherichia coli. J Gen Microbiol 74:77-91.

Azam F, Ammerman JW (1984) Cycling of organic matter by bacterioplankton in pelagic marine ecosystems: microenvironmental considerations. In: Fasham MJR (ed) Flows of energy and materials in marine ecosystems. Plenum Publishing. New York, p 345-360

Barbara GM, Mitchell JG (1996) Formation of 30-40-microm-

Editorial responsibility: Otto Kinne (Editor),

Oldendorf/Luhe, Germany eter-thick laminations by high-speed marine bacteria in microbial mats. Appl Environ Microbiol 62:3985-3990

Berg HC, Brown DA (1972) Chemotaxis in Escherichia coli analysed by three-dimensional tracking. Nature 239: 500-504

Blackburn N, Azam F, Hagström $\AA$ (1997) Spatially explicit simulations of a microbial food web. Limnol Oceanogr 42 : $613-622$

Blackburn N, Fenchel T, Mitchell J (1998) Microscale nutrient patches in planktonic habitats shown by chemotactic bacteria Science 282:2254-2256

Bowen JD, Stolzenback KD (1992) The concentration distribution near a continuous point source in steady homogeneous shear. J Fluid Mech 236:95-110

Bowen JD, Stolzenbach KD, Chisholm SW (1993) Simulating bacterial clustering around phytoplankton cells in a turbulent ocean. Limnol Oceanogr 38:36-51

Brown DA, Berg HC (1974) Temporal stimulation of chemotaxis in Escherichia coll. Proc Nat Acad Sci USA 71: $1388-1392$

Duarte CM, Vaqué D (1992) Scale dependence of bacterioplankton patchiness. Mar Ecol Prog Ser 84:95-100

Fenchel T, Glud RN (1998) Veil architecture in a sulphide-oxidizing bacterium enhances countercurrent flux. Nature 394:367-369

Itsweire EC, Osborn TR (1988) Microstructure and vertical velocity shear distribution in Monterey Bay. In: Nihoul JCJ, Jamart BM (eds) Small-scale turbulence and mixing in the ocean. Elsevier, Amsterdam, p 213-227

Jackson GA (1987) Simulating chemosensory responses of marine microorganisms. Limnol Oceanogr 32:1253-1266

Jumars PA, Deming JW, Hill PS, Karp-Boss L, Yager PL, Dade WB (1993) Physical constraints on marine osmotrophy in an optimal foraging context. Mar Microb Food Webs 7 : $121-159$

Koch AL, Wang CH (1982) How close to the theoretical diffusion limit do bacterial uptake systems function? Arch Microbiol 131:36-42

Krembs C, Juhl AR, Long RA, Azam F (1998) Nanoscale patchiness of bateria in lake water studied with the spatial information preservation method. Limnol Oceanogr 43 307-314

Lazier JRN, Mann KH (1989) Turbulence and the diffusive layers around small organisms. Deep-Sea Res 36: $1721-1733$

Mitchell JG, Pearson L, Dillon S (1996) Clustering of marine bacteria in seawater enrichments. Appl Environ Microbiol $62: 3716-3721$

Submitted: March 9, 1999; Accepted: June 8, 1999 Proofs received from author(s): November 1, 1999 